Grigorenko Elena E. (Orcid ID: 0000-0003-0670-4642)

Dubyagin Stepan (Orcid ID: 0000-0002-0888-2517)

Khotyaintsev Yu. V. (Orcid ID: 0000-0001-5550-3113)

Kronberg Elena A. (Orcid ID: 0000-0001-7741-682X)

Lavraud Benoit (Orcid ID: 0000-0001-6807-8494)

Ganushkina Natalia Yu (Orcid ID: 0000-0002-9259-850X)

\title{
Intense current structures observed at electron kinetic scales in the near-Earth magnetotail during dipolarization and substorm current wedge formation
}

E. E. Grigorenko ${ }^{1,2}$, S. Dubyagin ${ }^{3}$, A. Yu. Malykhin ${ }^{1}$, Yu. V. Khotyaintsev ${ }^{4}$, E. A. Kronberg $^{5,6}$, B. Lavraud ${ }^{7}$, N. Yu. Ganushkina ${ }^{3,8}$

${ }^{1}$ Space Research Institute of RAS, Moscow, Russia.

${ }^{2}$ Moscow Institute of Physics and Technology, Moscow, Russia.

${ }^{3}$ Finnish Meteorological Institute, Helsinki, Finland.

${ }^{4}$ Swedish Institute of Space Physics, Uppsala, Sweden

${ }^{5}$ Max Planck Institute for Solar System Research, Göttingen, Germany

${ }^{6}$ Ludwig Maximilian University of Munich, Munich, Germany

${ }^{7}$ Institut de Recherche en Astrophysique et Planétologie, UMR 5277, Université de Toulouse (UPS), CNRS, CNES, Toulouse, France

${ }^{8}$ University of Michigan, Ann Arbor, MI, USA

Corresponding author: Elena E. Grigorenko (elenagrigorenko2003@yandex.ru)

This is the author manuscript accepted for publication and has undergone full peer review but has not been through the copyediting, typesetting, pagination and proofreading process, which may lead to differences between this version and the Version of Record. Please cite this article as doi: 10.1002/2017GL076303

This article is protected by copyright. All rights reserved. 


\title{
Key points:
}

- Multiscale current structure formed during dipolarization growth

- Intense current structures are transiently $(\leq 2 s)$ observed at the leading and trailing edges of $B_{Z}$ pulses during dipolarization growth

- Spatial scales of the intense current structures are $\sim 100-200 \mathrm{~km} \sim 2.5-5.0 \lambda_{\mathrm{e}}$

\begin{abstract}
We use data from the 2013-2014 Cluster Inner Magnetosphere Campaign, with its uniquely small spacecraft separations ( $\leq$ electron inertia length, $\lambda_{\mathrm{e}}$ ), to study multiscale magnetic structures in 14 substorm-related prolonged dipolarizations in the near-Earth magnetotail. Three time scales of dipolarization are identified: i) a prolonged growth of the $B_{Z}$ component with duration $\leq 20 \mathrm{~min}$; ii) $B_{Z}$ pulses with durations $\leq 1$ min during the $B_{Z}$ growth; iii) strong magnetic field gradients with durations $\leq 2 \mathrm{~s}$ during the dipolarization growth. The values of these gradients observed at electron scales are several dozen times larger than the corresponding values of magnetic gradients simultaneously detected at ion scales. These nonlinear features in magnetic field gradients denote the formation of intense and localized ( a few $\left.\lambda_{\mathrm{e}}\right)$ current structures during the dipolarization and substorm current wedge formation. These observations highlight the importance of electron-scale processes in the formation of a 3D substorm current system.
\end{abstract}

This article is protected by copyright. All rights reserved. 


\section{Introduction}

The dipolarization of the Earth's magnetotail magnetic field, observed as an increase in the positive $B_{Z}$ component, is an essential element of substorm onset [e.g. Baumjohann et al., 1999; Sergeev et al., 2012 and references therein]. The source(s) of these perturbations is still debated. According to one widely discussed scenario, the development of plasma instabilities triggers current disruption in the near-Earth Plasma Sheet (PS) [Lui et al., 1991; Roux et al., 1991; Lui, 2004]. Another scenario considers magnetic reconnection-induced Bursty Bulk Flows (BBFs), which transport magnetic flux and energy to the inner magnetosphere and destabilize the near-Earth PS [e.g. Hayakawa et al., 1982; Baumjohann et al., 1990; Angelopoulos et al., 1992; 1994; Baker et al., 1996]. BBFs are accompanied by $B_{Z}$ enhancements - i.e., dipolarization fronts (DFs) which are spatial structures traveling with the flow. DFs are often associated with impulsive electric fields, wave bursts and enhancements of energetic particle fluxes [e.g. Ohtani et al., 2004; Runov et al., 2009; 2011; Zhou et al., 2009; Deng et al., 2010; Khotyaintsev et al., 2011; Ergun et al., 2014].

DFs have been studied extensively in the last decades. It was shown that they represent kinetic structures with vertical thin ( thermal ion gyroradius) Current Sheets (CSs) embedded within the BBF [e.g. Runov et al., 2009; Sergeev et al., 2009, Khotyaintsev et al., 2011; Fu et al., 2012]. Later studies showed that at smaller sub-ion 
gyroscales, DFs are made of complex and structured CSs that may contain small-scale dissipative layers [e.g. Angelopoulos et al., 2013; Balikhin et al., 2014].

In the transition region (the so-called flow braking region), where the stretched magnetotail-like magnetic configuration transforms to a more dipole-like shape (at $X_{\sim}$ $10 \mathrm{R}_{\mathrm{E}}$ ), BBFs are decelerated and oscillate [e.g. Shiokawa et al., 1997; Baumjohann et al., 2002; Panov et al., 2010; 2015]. This results in the formation of a magnetic flux pile-up region, perturbations of the near-Earth Plasma Sheet (PS) and a cross-tail current through the development of various plasma instabilities [e.g. Roux et al., 1991; Lui, 2004; Grigorenko et al., 2014 and references therein]. The perturbations of the CS are manifested in the development of long-lasting dipolarizations (tens of minutes), which can be preceded and/or comprised of multiple positive pulses in the $B_{Z}$ field.

The origin of these pulses has been debated to be either the passage of spatial magnetic structures - DFs/dipolarizing flux bundles (DFBs) [e.g. Runov et al., 2011; Nakamura et al., 2013; Liu et al., 2013; 2014; Gabrielse et al., 2014; 2017], which then pile-up in the near-Earth PS and cause a prolonged dipolarization - or signatures of a near-Earth instability that leads to a global dipolarization [e.g. Lui, 2004].

The reduction and/or diversion of the cross-tail electric current at macro-scale is manifested in a gradual growth of the $B_{Z}$ field [e.g. Lui, 2011]. The arrival and subsequent pile-up of multiple DFs with their own current systems [e.g. Liu et al., 2015] result in the formation of a multiscale 3D current pattern that contributes to the formation 
of a substorm current system. Knowledge of the electric current structure in this region and its evolution during the dipolarization is crucial for the understanding of the processes responsible for the formation of the substorm current wedge (SCW).

In the present paper, we use data from the Cluster Inner Magnetosphere Campaign, with its uniquely small spacecraft separations (down to a few $\mathrm{km}$ ), to study the magnetic structure of prolonged dipolarizations in the near-Earth PS (at $-15 \mathrm{R}_{\mathrm{E}} \leq X \leq$ $\left.7 R_{E}\right)$ associated with the arrival and braking of multiple BBFs and DFs. The very small separation between Cluster-3 and Cluster-4 permits, for the first time, the observation of strong magnetic gradients at electron scales, suggesting that intense and localized current structures form during prolonged dipolarization growths. These observations denote the importance of the processes occurring at electron scales in the formation of a $3 \mathrm{D}$ substorm current system.

\section{Observations}

In this study we used the Cluster magnetic field data collected by the fluxgate magnetometer (FGM) [Balogh et al., 2001] in both spin (4s) and high time resolution mode $(22.4 \mathrm{~Hz})$; electric field data (spin resolution mode) collected by the Electric Field and Wave (EFW) instrument [Gustafsson et al., 2001]; ion moments from the Composition and Distribution Function Analyser (CODIF) [Rème et al., 2001] and electron moments collected by the Plasma Electron and Current Experiment (PEACE) 
[Johnstone et al., 1997]. The GSM coordinate system is used for orbit, magnetic field and ion data.

\subsection{Overview of dipolarization in the near-Earth PS}

In this section we present an overview of the PS dynamics during a dipolarization event observed by the Cluster spacecraft in the postmidnight sector at $X \sim-9 \mathrm{R}_{\mathrm{E}}$ on 20 July 2013. This event is representative of 14 similar dipolarization events analyzed in section 3. Figure 1 shows an overview of this event. The location of Cluster is shown in the $(X Y)$ and $(Y Z)$ planes in the right part of the Figure. During the interval of interest, Cluster-3 (C3) and Cluster-4 (C4) had very close locations: $\Delta X_{\mathrm{C} 3-\mathrm{C} 4} \sim 6.5 \mathrm{~km} ; \Delta Y_{\mathrm{C} 3-\mathrm{C} 4} \sim 65$ $\mathrm{km} ; \Delta Z_{\mathrm{C} 3-\mathrm{C} 4} \sim 6.5 \mathrm{~km}$. The position of $\mathrm{C} 4$ relative to $\mathrm{C} 3$ is shown in two bottom panels in the right part of Figure 1.

The growth of the dipolarization was observed between 01:37 and 01:50 UT along with a decrease in the $A L$ index (see Figure 1a,c). After reaching a dipolarized state, the $B_{Z}$ value remained large. During the entire interval, Cluster-1 (C1), C3 and C4 were located in the northern PS in the region with $\left|B_{X}\right| \leq 10$ nT (see Figure 1d). C2 was initially located in the outer part of the southern PS (at $B_{X} \sim-20 \mathrm{nT}$ ) and it was gradually approaching the equatorial plane as the dipolarization progressed.

The onset of dipolarization is manifested in a sharp increase of the positive $B_{Z}$ field (DF) observed at 01:37:20 UT by C1 (marked by a vertical black dashed line), 
then by the pair C3-C4 at 01:37:35 UT (marked by a vertical magenta dashed line). At the location of $\mathrm{C} 2$, the gradual growth of the $B_{Z}$ field started after 01:37:40 UT (marked by a red vertical line) without a pronounced DF. This is possibly because C2 was located in the outer PS at the beginning of the event.

This event displays typical features reported in many earlier studies [e.g. Apatenkov et al., 2007; Ge et al., 2011; Baumjohann et al., 1999; Birn et al., 2011; Kronberg et al., 2017]. The onset of dipolarization coincides with the beginning of the decrease of $A L$, indicating the formation of the SCW (see Figure 1a). At the location of Cluster, the onset-related DF was observed along with a tailward flow (see Figure 1e). At this time, the THEMIS P3 probe was also located in the northern PS at $X \sim-8.5 \mathrm{R}_{\mathrm{E}}$ but $\sim 3 \mathrm{R}_{\mathrm{E}}$ duskward of Cluster (not shown). The P3 probe observed the onset-related DF along with the arrival of earthward moving $\mathrm{BBF} \sim 1 \mathrm{~min}$ earlier than at $\mathrm{C} 1$. This indicates that Cluster was located near the dawn side of the BBF channel and close to the flow braking region, thus detecting a reflected/diverted or vortical flow feature [e.g. Keika et al., 2009; Keilling et al., 2009; Panov et al., 2010; Birn et al., 2011].

The dawnward diversion of the onset-related DF is evident from the time sequence of its observations by the THEMIS P3 probe and different Cluster satellites. It is also confirmed by the normal directions $(N)$ deduced from the minimum variance analysis (MVA) [Sönnerup and Scheible, 1998] applied to this DF observed by P3, C1, $\mathrm{C} 3$ and $\mathrm{C} 4: \quad N_{\mathrm{P} 3}=[0.9,-0.2,-0.35], \quad N_{\mathrm{C} 1}=[-0.6,-0.6,0.5], \quad N_{\mathrm{C} 3, \mathrm{C} 4}=[-0.2,-0.8,0.5] . \quad$ The 
azimuthal deflection of the DF also confirms that Cluster was located near the flow braking region [e.g. Ge et al., 2011].

After the dipolarization onset, two enhancements of positive $V_{X}$ were observed between 01:38 UT and 01:42 UT, indicating the arrival of BBFs at Cluster's location (see Figure 1e). Later, after 01:42 UT, the $V_{X}$ experienced negative and positive variations indicating oscillations of the flux tubes [e.g. Panov et al., 2015 and references therein].

During the gradual growth of the $B_{Z}$ field at 01:37-01:50 UT, short positive pulses of $B_{Z}$ with durations $\sim$ tens of seconds were detected by Cluster (see Figure 1c). The strongest $B_{Z}$ pulse with an amplitude of $\Delta B_{Z} \sim 10 \mathrm{nT}$ was detected by all Cluster satellites except C2 around 01:40 UT. The pulse propagated dawnward with $V_{Y} \sim-400 \mathrm{~km} / \mathrm{s}$ as estimated by a timing analysis of the high-resolution magnetic field observations. Just after the pulse, there is a strong increase of positive $V_{X}$ up to $\sim 600 \mathrm{~km} / \mathrm{s}$, indicating the arrival of the fast earthward flow at Cluster's location (see Figure 1e). Simultaneously with the flow burst at 01:39:45 UT, a pulse of the dawn-to-dusk electric field $\left(E_{Y}\right)$ with an amplitude of $\sim 35 \mathrm{mV} / \mathrm{m}$ and a drop in the electron density were detected (see Figure 1f,g). This interval is shaded in pink in Figure 1.

Figure $1 \mathrm{~h}$ displays the time profiles of $\Delta B_{X} / \Delta Y_{[\mathrm{C} 3-\mathrm{C} 4]}$ calculated between the closely spaced $\mathrm{C} 3$ and $\mathrm{C} 4$ by using high-resolution magnetic field data. As will be shown below, the normals $(\mathbf{N})$ to the magnetic structures, which are associated with strong gradients, are directed mostly along the $\mathbf{Y}$ axis while the direction of maximum magnetic 
field variation (L) is almost along the $\mathbf{X}$ axis (that is, $\Delta B_{X} / \Delta Y_{[\mathrm{C} 3-\mathrm{C} 4]} \sim \Delta B_{L} / \Delta N_{[\mathrm{C3}-\mathrm{C} 4]}$ ). The gray profile shows $\Delta B_{X} / \Delta Y_{[\mathrm{C} 3-\mathrm{C} 4]}$ including the background level, while the black profile shows only $\Delta B_{X} / \Delta Y_{[\text {[C3-C4] }}$ values which exceed the background amplitude. $\left|\Delta B_{X} / \Delta Y_{[\mathrm{C3} 3 \mathrm{C} 4]}\right|$ values below the background cutoff value are set to zero. The background cutoff value was estimated by averaging $\mid \Delta B_{X} / \Delta Y_{\text {[C3-C4] }]}$ for two minutes before the dipolarization onset (at 01:35-01:37 UT).

The $\Delta B_{X} / \Delta Y_{[\mathrm{C} 3-\mathrm{C} 4]}$ values which contribute to the electric current density $J_{Z}$ experience the strongest bipolar variations during the pink shaded interval (see Figure 1). For comparison, in Figure 1i we present $\Delta B_{X} / \Delta Y_{[\mathrm{C} 1-\mathrm{C} 4]}$ calculated between $\mathrm{C} 1$ and $\mathrm{C} 4$. The amplitude of $\Delta B_{X} / \Delta Y_{[\mathrm{Cl}-\mathrm{C} 4]}$ variations is up to 100 times smaller than the amplitude of $\Delta B_{X} / \Delta Y_{[\mathrm{C} 3-\mathrm{C} 4]}$ variations. This indicates the transient generation of a strong magnetic field gradient between $\mathrm{C} 3$ and $\mathrm{C} 4$ (i.e. at the spatial scale $\sim 65 \mathrm{~km}$ ). Below we present a detailed analysis of the associated current structures as observed by $\mathrm{C} 3$ and $\mathrm{C} 4$.

\subsection{Analysis of current structures associated with strong magnetic gradients}

In the left part of Figure 2, we show a zoom of the pink shaded interval shown in Figure 1 (01:39-01:40:20 UT). In this interval, a pulse in $B_{Z}$ is observed along with an increase in the ion $V_{X}$, a decrease in the electron density and a positive pulse in the $E_{Y}$ field. The strongest $\Delta B_{X} / \Delta Y_{\mathrm{C} 3-\mathrm{C} 4}$ variations (with amplitudes $>20 \mathrm{nT} / 1000 \mathrm{~km}$, see gray shaded intervals in Figure 2) are detected at the leading and trailing edges of the $B_{Z}$ pulse. 
The first strong bipolar variation in $\Delta B_{X} / \Delta Y_{\mathrm{C} 3-\mathrm{C} 4}$ was observed in the $2 \mathrm{~s}$ interval 01:39:37-01:39:39 UT (interval "I"), at the leading edge of the $B_{Z}$ pulse and the highspeed plasma flow, just before a positive pulse in the dawn-dusk electric field $E_{Y}$ (see Figure 2a,d,e). Unfortunately, for electric field and plasma moments we only have 4s time resolution data. Thus, we cannot perform an exact timing analysis of the relationship between the bursty appearance of strong magnetic gradients and the dynamics of electric field and plasma characteristics.

The amplitude of the positive $\Delta B_{X} / \Delta Y_{\mathrm{C} 3-\mathrm{C} 4}$ pulse reached $57 \mathrm{nT} / 1000 \mathrm{~km}$. During this $2 \mathrm{~s}$ interval, the strongest difference between the magnetic field components measured by $\mathrm{C} 3$ and $\mathrm{C} 4$ was observed for the $B_{X}$ field. The difference between the other components was significantly smaller: $\Delta B_{Y[C 3-C 4]}<<\Delta B_{X[C 3-C 4]}$ and $\Delta B_{Z[C 3-C 4]} \sim 0$ nT. Since the electric current density $J_{Z}$ is proportional to $\Delta B_{X} / \Delta Y: J_{Z} \sim \mu_{0}{ }^{-1} \cdot \Delta B_{X} / \Delta Y$, we surmise that the transient enhancement in $\Delta B_{X} / \Delta Y$ denotes the crossing of a current structure (possibly a current filament) with an intense $J_{Z}$. The negative part of the bipolar $\Delta B_{X} / \Delta Y_{\mathrm{C} 3-\mathrm{C} 4}$ variation has much smaller amplitude.

The second enhancement in the $\left|\Delta B_{X} / \Delta Y_{\mathrm{C} 3-\mathrm{C} 4}\right|$ (interval "II") was observed at 01:39:50-01:39:55 UT. During this interval, only positive variations of $\Delta B_{X} / \Delta Y_{\mathrm{C} 3-\mathrm{C} 4}$ with amplitudes of up to $\sim 53 \mathrm{nT} / 1000 \mathrm{~km}$ were observed. By the end of this interval, the value of the positive $\Delta B_{X} / \Delta Y_{\mathrm{C} 3-\mathrm{C} 4}$ decreased to zero. 
The third strong bipolar variation of $\Delta B_{X} / \Delta Y_{\mathrm{C} 3-\mathrm{C} 4}$ (interval "III") was observed immediately after the second one at 01:39:55-01:39:59 UT. Both "II" and "III" enhancements in $\Delta B_{X} / \Delta Y_{\mathrm{C} 3-\mathrm{C} 4}$ took place at the trailing edge of the high-speed plasma flow and the $B_{Z}$ pulse and after the pulse in $E_{Y}$.

To estimate, at least partially, the spatial scales of the electric current structures associated with the $\Delta B_{X} / \Delta Y_{\mathrm{C} 3-\mathrm{C} 4}$ bursts we applied the MVA analysis to high resolution magnetic field data observed during the intervals "I"-“III". In all three intervals, C3 and C4 observed similar variations in $B_{X}$, suggesting that a spatial structure crossed the positions of $\mathrm{C} 3$ and $\mathrm{C} 4$.

For interval I (01:39:37.300-01:38:00.100 UT), the directions of $\mathbf{N}$ at the position of $\mathrm{C} 3$ and $\mathrm{C} 4$ are very similar: $\mathbf{N}_{\mathrm{C} 3}=[-0.4,0.86,0.3] ; \mathbf{N}_{\mathrm{C} 4}=[-0.5,0.8,0.3]$. The eigenvalue ratios are $\lambda_{2} / \lambda_{1} \sim 12$ and $\lambda_{3} / \lambda_{2} \sim 9$. We estimated the propagation velocity ( $\left.V_{\text {prop }}\right)$ of the current structure along $\mathbf{N}$ using time delays in the $B_{X}$ variations observed by $\mathrm{C} 3$ and $\mathrm{C} 4$. The estimate is $V_{\text {prop }} \sim 400 \mathrm{~km} / \mathrm{s}$ and the direction is mainly dawnward.

For interval II (01:39:49.600-01:39:53.900 UT), we obtained $\mathbf{N}_{\mathrm{C} 3}=[-0.5,0.84,0.2]$, $\mathbf{N}_{C 4}=[-0.5,0.86,0.2]$, and $\lambda_{2} / \lambda_{1} \sim 9, \lambda_{3} / \lambda_{2} \sim 6$. In this interval, $V_{\text {prop }}$ decreased to $300 \mathrm{~km} / \mathrm{s}$ and changed its direction to duskward.

For interval III (01:39:55.400-01:39:59.400 UT), we defined $\mathbf{N}_{\mathrm{C} 3}=[-0.4,0.8,0.5]$, $\mathbf{N}_{\mathrm{C} 4}=[-0.4,0.75,0.5]$ and $\lambda_{2} / \lambda_{1} \sim 8.5, \lambda_{3} / \lambda_{2} \sim 7$. For this interval, $V_{\text {prop }} \sim 180 \mathrm{~km} / \mathrm{s}$ and the direction is dawnward. 
Thus, for all three intervals of strong magnetic gradients, the $\mathbf{N}$ has similar directions at $\mathrm{C} 3$ and $\mathrm{C} 4$ and it is mainly directed along $\mathbf{Y}$. The changes in the direction of $V_{\text {prop }}$ are more or less consistent with the changes in the sign of the proton velocity $V_{Y}$ as observed by the CODIF instrument onboard C4 (see Figure 2d). Some delay between the $V_{Y}$ and $V_{\text {prop }}$ reversals may be due to the difference in time resolutions between magnetic field and ion data. These changes in magnitude and direction of $V_{\text {prop }}$ indicate the oscillation and braking of the magnetic flux tubes.

In the right part of Figure 2, we present spatial profiles of the electric current $J_{Z}{ }^{*} \sim \mu_{0}^{-1} \cdot \Delta B_{X} / \Delta Y_{\mathrm{C} 3-\mathrm{C} 4}$ versus the coordinate $l$, which was calculated for each time $t_{\mathrm{i}}$ within intervals I-III as $l=V_{\text {prop }} \cdot t_{\mathrm{i}}$, and then shifted so that the zero $l$-value corresponds to the maximum of $J_{Z}{ }^{*}$ observed in the given time interval.

During interval "I", the bipolar variation of $\Delta B_{X} / \Delta Y_{\mathrm{C} 3-\mathrm{C} 4}$ provides the corresponding bipolar structure in the $J_{Z}{ }^{*}$ current. The half-thickness of the positive current $J_{Z I}{ }^{*}$ is $L_{J 1} \sim 100 \mathrm{~km}$ or $\sim 3 \lambda_{\mathrm{e}}$ (here $\lambda_{\mathrm{e}} \sim 37 \mathrm{~km}$ is the electron inertia length at the time of the positive $\Delta B_{X} / \Delta Y_{\mathrm{C} 3-\mathrm{C} 4}$ burst). The half-thickness of the negative current structure is $L_{J 2} \sim 150 \mathrm{~km} \sim 3 \lambda_{\mathrm{e}}\left(\lambda_{\mathrm{e}} \sim 47 \mathrm{~km}\right)$. The absolute values of the $J_{Z}{ }^{*}$ current density are $46 \mathrm{nA} / \mathrm{m}^{2}$ and $25 \mathrm{nA} / \mathrm{m}^{2}$, respectively. The total 3D current density may be even larger.

For interval "II", we obtain a more complicated shape of the $J_{Z}^{*}$ profile, consisting of four bursts (or filaments) of $J_{Z}$. The spatial scales $L$ of these filaments are $L_{J 1} \sim 200 \mathrm{~km}$ 
$\left(\sim 4.5 \lambda_{\mathrm{e}}\right) ; L_{J 2} \sim 150 \mathrm{~km}\left(\sim 3 \lambda_{\mathrm{e}}\right) ; L_{J 3} \sim 170 \mathrm{~km}\left(\sim 3.5 \lambda_{\mathrm{e}}\right)$ and $L_{J 4} \sim 100 \mathrm{~km}\left(\sim 2 \lambda_{\mathrm{e}}\right)$. The current density $J_{Z}^{*}$ ranges from $\sim 24 \mathrm{nA} / \mathrm{m}^{2}$ to $\sim 43 \mathrm{nA} / \mathrm{m}^{2}$.

During interval "III", the bipolar current structure is observed again. The spatial scale of the negative $J_{Z}{ }^{*}$ current is $L_{\mathrm{J} 1} \sim 200 \mathrm{~km} \sim 5 \lambda_{\mathrm{e}}\left(\lambda_{\mathrm{e}} \sim 40 \mathrm{~km}\right)$. Therein, a thinner current with $L \sim 100 \mathrm{~km} \sim 2.5 \lambda_{\mathrm{e}}$ is embedded. The peak current density in this structure is $\sim 65$ $\mathrm{nA} / \mathrm{m}^{2}$. The spatial scale of the positive $J_{Z}{ }^{*}$ current is $L_{\mathrm{J} 2} \sim 120 \mathrm{~km} \sim 3 \lambda_{\mathrm{e}}$ and the density is $J_{Z}{ }^{*} \sim 30 \mathrm{nA} / \mathrm{m}^{2}$. It is worth noting that the sign change in $V_{\text {prop }}$ is observed just at the beginning of interval III. The reversal motion of the flux tube along with the change in $J_{Z}$ * may be interpreted as a temporal variation in the electric current. However, since we cannot estimate the total 3D current density and its direction, we cannot determine if this variation is really caused by a fast $(\leq 1 \mathrm{~s})$ reconfiguration of the electric current structures at the trailing edge of the $B_{Z}$ pulse.

\section{Statistical study of strong magnetic field gradients during dipolarizations}

During the entire magnetotail season of the 2013-2014 Cluster Inner Magnetosphere Campaign, we found 13 additional dipolarization events similar to the one discussed in section 2. During all these events, Cluster was located in the PS at $-15 R_{E}$ $\leq X \leq-7 \mathrm{R}_{\mathrm{E}}$ and had similar satellite configurations: the separation of $\mathrm{C} 3$ and $\mathrm{C} 4$ was of the order of the electron inertial length or less $\left(\left|\Delta X_{\mathrm{C} 3-\mathrm{C} 4}\right|^{\text {median }} \sim 30 \mathrm{~km} ;\left|\Delta Y_{\mathrm{C} 3-\mathrm{C} 4}\right|^{\text {median }} \sim 40\right.$ $\mathrm{km} ;\left|\Delta Z_{\mathrm{C} 3-\mathrm{C} 4}\right|^{\text {median }} \sim 7 \mathrm{~km}$ ), while the separation between $\mathrm{C} 1$ and $\mathrm{C} 4$ was $\sim 1-2$ ion inertia lengths. 
The dates of the events are listed in the in the Supplementary materials, along with the maximum values of the $A L$ index $\left(|A L|_{\max }\right)$ and the maximum values of the $B_{Z}$ component $\left(B_{Z \max }\right)$ measured during each dipolarization event.

All dipolarizations in our data base, except for the one on 10/09/2013, were associated with geomagnetic perturbations and a significant decrease in the $A L$ index. The magnetic structure of the dipolarizations exhibited features similar to those discussed in the previous section. The duration of the prolonged $B_{Z}$ growth for the events ranges from a few minutes to $\sim 20 \mathrm{~min}$. Multiple short duration $(\leq 1 \mathrm{~min}) B_{Z}$ pulses were observed during the $B_{Z}$ growth for all events in our list.

To determine the statistical properties of these magnetic field gradients, we first applied the analysis described in section 2 to each event and identified the short intervals during which $\Delta B_{X} / \Delta Y_{[\mathrm{C} 3-\mathrm{C} 4]}$ exceeded a background value. We then applied MVA analysis to these intervals in order to calculate $\Delta B_{L} / \Delta N_{\text {[C3-C4] }}$. For further analysis of each (typically short, as discussed next) magnetic field gradient interval, the following conditions had to be satisfied: 1) MVA vectors calculated at $\mathrm{C} 3$ and $\mathrm{C} 4$ within $20^{\circ}$ from each other, 2) eigenvalue ratios $\lambda_{2} / \lambda_{1}$ and $\lambda_{3} / \lambda_{2} \geq 6.0$, and 3) a normal (N) along the direction of the largest C3-C4 separation (Y or X for our events). All intervals of strong magnetic gradients that did not fulfill these conditions were excluded from the statistical analysis described next. 
In all events, the strongest values of $\Delta B_{L} / \Delta N_{[\mathrm{C} 3-\mathrm{C} 4]}$ were observed during the dipolarization growth near the leading and trailing edges of the $B_{Z}$ pulses. The normals to these pulses were oriented mainly in the $(X Y)$ plane and condition (3) was almost always fulfilled.

To determine the phase of dipolarization during which the strong magnetic gradients are generated, we performed a superposed epoch analysis. The analysis was applied to the following parameters: $B_{Z} *, \Delta B_{L} / \Delta N_{[\mathrm{C} 3-\mathrm{C} 4]}, \Delta B_{L} / \Delta N_{[\mathrm{Cl} \text {-C4] }}$ calculated simultaneously with $\Delta B_{L} / \Delta N_{\text {[C3-C4] }}$, and the $A L^{*}$ index. Here, $B_{Z} *$ and $A L^{*}$ are the values of the $B_{Z}$ field and the $A L$ index observed in each event and normalized to the maximum values $B_{Z \max }$ and $|A L|_{\max }$, respectively (see the list of events presented above). As the epoch time $(t=0)$, we use the dipolarization onset for each event. To determine the onset we used the method described in Grigorenko et al. [2016].

Figure 3a-d shows the resulting superposed epoch profiles. Lower and upper quartiles are displayed in light green. The decrease in $A L$ index to its minimum value starts almost simultaneously with the dipolarization onset (at $t=0$ ) and ends $\sim 17$ min after the onset (this period is shaded pink in Figure 3a-d). During this period, the magnetic gradients $\left|\Delta B_{L} / \Delta N_{[\mathrm{C} 3-\mathrm{C} 4]}\right|$ and the corresponding electric current density $\left|J_{M}{ }^{*}\right|$, as estimated between $\mathrm{C} 3$ and $\mathrm{C} 4$ at small electron-type scales, transiently increased up to a few hundred $\mathrm{nA} / \mathrm{m}^{2}$ (see Figure 3b). The absolute values of electric current density $\left|J_{M}{ }^{*}\right|$, calculated as $\left|J_{M}{ }^{*}\right| \sim \mu_{0}{ }^{-1} \cdot\left|\Delta B_{L} / \Delta N_{\mathrm{C} 3-\mathrm{C} 4,}\right|$ are scaled according to the right vertical axis of 
Figure 3b. By contrast, at ion scales (as estimated between $\mathrm{C} 1$ and $\mathrm{C} 4$ according to their larger separation), the values of $\Delta B_{L} / \Delta N_{[\mathrm{Cl} \text {-C4] }}$ and the corresponding values of $\left|J_{M}{ }^{*}\right|$ are much smaller (see Figure 3c).

Figure $3 \mathrm{e}$ shows a histogram of the occurrence frequency of strong $\left|\Delta B_{L} / \Delta N_{\mathrm{C} 3-\mathrm{C} 4}\right|$ gradients $\left(\geq 25 \mathrm{nT} / 1000 \mathrm{~km}\right.$, that corresponds to $\left.\left|J_{M}{ }^{*}\right| \geq 20 \mathrm{nA} / \mathrm{m}^{2}\right)$ with a given duration. The occurrence frequency was calculated as the ratio of the number of strong gradients having durations within a given bin to the total number of strong gradients observed for all events. The majority of strong enhancements in $\left|\Delta B_{L} / \Delta N_{\mathrm{C} 3-\mathrm{C} 4}\right|$ are very short, with durations $\sim 0.1 \mathrm{~s}$.

Figure $3 f$ displays a histogram of the probability distribution of magnetic gradients $\left|\Delta B_{L} / \Delta N_{\mathrm{C} 3-\mathrm{C} 4}\right|$ and the corresponding electric current density $\left|J_{M}{ }^{*}\right|$. Values of $\left|\Delta B_{L} / \Delta N_{\mathrm{C} 3-\mathrm{C} 4}\right|$ are binned according to the bottom horizontal axis, and the corresponding values of $\left|J_{M}{ }^{*}\right|$ are given in the upper horizontal axis. The probability was calculated as the ratio of the total duration of $\left|\Delta B_{L} / \Delta N_{\mathrm{C} 3-\mathrm{C} 4}\right|$ (and $\left|J_{M}{ }^{*}\right|$ ) within a given range of values to the total duration of all dipolarizations. The observation probability is very small and decreases rapidly as $\Delta B_{L} / \Delta N_{\mathrm{C} 3-\mathrm{C} 4}$ and $\left|J_{M}{ }^{*}\right|$ increase.

\section{Discussion and Conclusions}

The small separation between the C3 and C4 spacecraft, which was achieved in the near-Earth PS during the Cluster Inner Magnetosphere Campaign in 2013-2014, 
permitted the observation of strong, transient magnetic gradients generated at electron scales at the leading and trailing edges of the $B_{Z}$ pulses during the prolonged dipolarization growth. This finding is obtained from the analysis of 14 such dipolarization events associated with the arrival of multiple BBFs with DFs.

Three time scales in the dipolarization evolution are identified: i) a prolonged growth of the $B_{Z}$ component with duration $\leq 20 \mathrm{~min}$; ii) $B_{Z}$ pulses with durations $\leq 1 \mathrm{~min}$ observed during the $B_{Z}$ growth; iii) strong and fast variations of $\Delta B_{L} / \Delta N(\leq 2 \mathrm{~s})$ at the leading and trailing edges of the $B_{Z}$ pulses. These strong enhancements in $\Delta B_{L} / \Delta N$ show that the formation of intense and localized current structures, with current densities up to a hundred $\mathrm{nA} / \mathrm{m}^{2}$, are frequent and typical during dipolarization growth.

The majority of strong magnetic gradients with amplitude $\Delta B_{L} / \Delta N_{\mathrm{C} 3-\mathrm{C} 4} \geq 25$ $\mathrm{nT} / 1000 \mathrm{~km}$ are very short, with durations less than $1 \mathrm{~s}$. For typical values of the structures propagation velocities obtained from our analysis $(\sim 100-400 \mathrm{~km} / \mathrm{s})$, these durations imply spatial scales for the current structures lower than a few hundred km, i.e. $\sim$ a few $\lambda_{\mathrm{e}}$

Multiple, transient $B_{Z}$ pulses during gradual dipolarization growth in the nearEarth PS were reported in many previous studies [e.g. Nakamura et al., 2009; Liu et al., 2013, 2014; Grigorenko et al., 2016; Gabrilese et al., 2017]. The associated current system was shown to contribute to the formation of the 3D substorm current system [e.g. Sergeev et al., 2012; Liu et al., 2013]. Our superposed epoch analysis further 
demonstrates a good agreement between the decrease in the $A L$ index, the prolonged growth of the $B_{Z}$ field and the transient appearance of strong and short magnetic gradients denoting the generation of intense electric currents at electron scales.

From such two-spacecraft data analysis we cannot determine the total current density and the direction of the electric current in these structures. However, our analysis (assuming a $1 \mathrm{D}$ current structure) showed that the observed $\Delta B_{L} / \Delta N$ typically mainly contributes to the $J_{Z}$ current density. Also, since after the dipolarization onset $|B| \sim B_{Z}$, the $J_{Z}$ current is almost field-aligned.

This finding complements recent MMS observations of strictly localized ( a few tens of km) and short-lived field-aligned currents observed in the Plasma Sheet Boundary Layer (PSBL) during a substorm [Nakamura et al., 2016]. These PSBL currents may represent the high-latitude extensions of the intense, transient current structures generated at electron scale in the deep PS, as reported in the present study.

Since field-aligned currents generated in the near-Earth PS in the course of dipolarization can contribute to the 3D substorm current system, the origin of these currents is an important question. Do they represent "short-lived" structures, which dissipate within seconds, or do they represent propagating spatial structures which are observed only for a short time because of their highly localized nature?

The results of our analysis rather support a highly localized nature. The spatial scales of the strong magnetic gradients and associated electric current structures are much 
smaller than the spatial scales of magnetic fluctuations expected from the development of, e.g., cross-field current instability or the shear flow ballooning instability in the nearEarth PS [e.g. Kalmoni et al., 2015; Lui, 2016]. The close similarity between the magnetic fluctuations observed at each Cluster spacecraft, as well as the similar propagation velocities of the fluctuations $\left(V_{\text {prop }}\right)$ and corresponding component of plasma bulk velocity, all point to a spatial nature. This is consistent with the resemblance reported between $V_{\text {prop }}$ values and the propagation velocities of DFs in previous works [e.g. Runov et al., 2009; Nakamura et al. 2009]. All these arguments support the hypothesis that these intense electric currents are part of a multiscale electric current system associated with DFs.

In conclusion, our observations show the importance of processes occurring at electron scales during the prolonged dipolarization growth in formation of the 3D substorm current system. This phenomenon requires further detailed studies using multipoint magnetic field observations at sub-ion spatial scales with high time resolution.

\section{Acknowledgements}

- We acknowledge Cluster Science Archive (CAA, http://www.cosmos.esa.int/web/csa), PI and teams of FGM, EFW, CIS and PEACE instruments for providing the data. The Cluster data were downloaded from CAA at http://www.cosmos.esa.int/web/csa.

This article is protected by copyright. All rights reserved. 
- The $A L$ data were downloaded from the WDC for Geomagnetism, Kyoto at http://wdc.kugi.kyoto-u.ac.jp/aeasy/index.html.

- We are grateful to V.A. Sergeev for his very useful comments and suggestions.

- The work of E.E. Grigorenko and A.Yu. Malykhin was supported by RSF (grant \#14-12-00824).

- E. A. Kronberg acknowledges the "Deutsches Zentrum für Luft und Raumfahrt (DLR)" (grant 50 OC 1602).

- Work at IRAP was supported by CNRS and CNES.

- The research of N. Ganushkina and S. Dubyagin was partly funded by the European Union's Horizon 2020 research and innovation program (grant No. 637302 PROGRESS). N. Ganushkina acknowledges research grants from NASA (NNX14AF34G, NNX17AI48G).

\section{References}

Angelopoulos, V., Baumjohann, W., Kennel, C. F., Coroniti, F. V., Kivelson, M. G., Pellat, R., Walker, R. J., Lühr, H., and Paschmann, G. (1992), Bursty bulk flows in the inner central plasma sheet, J. Geophys. Res., 97, 4027-4039.

Angelopoulos V., et al. (1994), Statistical characteristics of bursty bulk flow events, J. Geophys. Res., 99, 21257-21280, doi:10.1029/94JA01263.

This article is protected by copyright. All rights reserved. 
Angelopoulos V., A. Runov, X.-Z. Zhou, D.L. Turner, S.A. Kiehas, S.-S. Li, I. Shinohara (2013), Electromagnetic energy conversion at reconnection front, Science, 341, 1478-1482, doi:10.1126/science.1236992.

Apatenkov, S. V., et al. (2007), Multi-spacecraft observation of plasma dipolarization/injection in the inner magnetosphere, Ann. Geophys., 25, 801-814, doi:10.5194/angeo-25-801-2007.

Baker D.N., T.I. Pulkkinen, V. Angelopoulos, W. Baumjohann, R.L. McPherron (1996), Neutral line model of substorms: Past results and present view, J. Geophys. Res., 101, 12975-13010, doi:10.1029/95JA03753.

M.A. Balikhin, A. Runov, S.N. Walker, M. Gedalin, I. Dandouras, Y. Hobara, A. Fazakerley (2014), On the fine structure of dipolarization fronts, J. Geophys. Res., 119, 6367-6385, doi:10.1002/2014JA019908.

Balogh, A., et al. (2001), The Cluster magnetic filed investigation: Overview of inflight performance and initial results, Ann. Geophys., 19, 1207-1217.

Baumjohann W., G. Paschmann, H. Luehr (1990), Characteristics of high-speed ion flows in the plasma sheet, J. Geophys. Res., 95, 3801-3809, doi:10.1029/JA095iA04p03801.

Baumjohann W., M. Hesse, S. Kokubun, T. Mukai, T. Nagai, A.A. Petrukovich (1999), Substorm dipolarization and recovery, J. Geophys. Res., 104, 24,995-25,000, doi:10.1029/1999JA900282.

This article is protected by copyright. All rights reserved. 
Baumjohann W. (2002), Modes of convection in the magnetotail, Phys. Plasmas, 9, 3665-3667, doi:10.1063/1.499116.

Birn J., R. Nakamura, E.V. Panov, M. Hesse (2011), Bursty bulk flows and dipolarization in MHD simulations of magnetotail reconnection, J. Geophys. Res., 116, A01210, doi:10.1029/2010JA016083.

Deng X., M. Ashour-Abdalla, M. Zhou, R. Walker, M. El-Alaoui, V. Angelopoulos, R.E. Ergun, D. Schriver (2010), J. Geophys. Res., 115, A09225, doi:10.1029/2009JA015107.

R. E. Ergun, K. A. Goodrich, J. E. Stawarz, L. Andersson, V. Angelopoulos (2014), Large-amplitude electric fields associated with bursty bulk flow braking in the Earth's plasma sheet, J. Geophys. Res., 120, 1832-1844, doi:10.1002/2014JA020165.

H. S. Fu, Y.V. Khotyaintsev, A. Vivads, M. André, S.Y. Huang (2012), Electric structure of dipolarization front at sub-proton scale, Geophys. Res. Lett., 39, L06105, doi:10.1029/2012GL051274.

Gabrielse C., V. Angelopoulos, A. Runov and D. L. Turner (2014), Statistical characteristics of particle injections throughout the equatorial magnetotail, J. Geophys. Res., 119, 2512-2535, doi:10.1002/2013JA019638.

Gabrielse, C., V. Angelopoulos, C. Harris, A. Artemyev, L. Kepko, and A. Runov (2017), Extensive electron transport and energization via multiple, localized dipolarizing flux bundles, J. Geophys. Res. Space Physics, 122, doi:10.1002/2017JA023981.Y.S. Ge, 
J. Raeder, V. Angelopoulos, M.L. Gilson, A. Runov (2011), Interaction of dipolarization fronts within multiple bulk flows in global MHD simulations of a substorm on 27 February 2009, J. Geophys. Res., 116, A00123, doi:10.1029/2010JA015758.

Grigorenko E.E., J.-A. Sauvaud, L. Palin, C. Jacquey, L.M. Zelenyi (2014), THEMIS observations of the Current Sheet dynamics in response to the intrusion of the high-velocity plasma flow into the near-Earth magnetotail, J. Geophys. Res., 119, doi:10.1002/2013JA019729.

E. E. Grigorenko, E. A. Kronberg, P. W. Daly, N. Yu. Ganushkina, B. Lavraud, J.- A. Sauvaud, L. M. Zelenyi (2016), Origin of low proton-to-electron temperature ratio in the Earth's plasma sheet, J. Geophys. Res., Space Physics, 121, doi:10.1002/2016JA022874.

Gustafsson G. et al. (2001), First results of electric field and density observations by CLUSTER EFW based on initial months of operation, Ann. Geophys., 19, 1219-1240.

Hayakawa H.A., A. Nishida, E.W. Hones Jr, S.J. Bame (1982), Statistical characteristics of plasma flow in the magnetotail, J. Geophys. Res., 87, 277-283, doi:10.1029/JA087iA01p00277.

Johnstone, A.D., et al. (1997), PEACE: A plasma electron and current experiment, Space Sci. Rev., 79, 351-398.

This article is protected by copyright. All rights reserved. 
N.M.E. Kalmoni, L.J. Rae, C.E.J. Watt, K.R. Murphy, C. Forsyth and C.J. Owen (2015), Statistical characterization of the growth and spatial scales of the substorm onset arc, J. Geophys. Res., 120, 8503-8516, doi:10.1002/2015JA021470.

Keika K., et al. (2009), Observations of plasma vorticies in the vicinity of flowbraking: A case study, Ann. Geophys., 27, 3009-3017, doi:10.5194/angeo-27-3009-2009.

Keilling A., et al. (2009), Substorm current wedge driven by plasma flow vortices: THEMIS observations, J. Geophys. Res., 114, A00C22, doi:10.1029/2009JA014114.

Khotyaintsev Yu.V., C. M. Cully, A. Vaivads, and M. André (2011), Plasma Jet Braking: Energy Dissipation and Nonadiabatic Electrons, PRL, 106, DOI: 10.1103/PhysRevLett.106.165001.

E. Kronberg, E. Grigorenko, D. Turner, P. Daly, Yu. Khotyaintsev (2017), Comparing and contrasting dispersionless injections at geosynchronous orbit during a substorm event, J. Geophys. Res., 122, 3, 3055-3072, doi:10.1002/2016JA023551.

Liu J., V. Angelopoulos, A. Runov and X.-Z. Zhou (2013), On the current sheets surrounding dipolarizing flux bundles in the magnetotail. The case of wedgelets, J. Geophys. Res., 118, 2000-2020, doi:10.1002/jgra.50092.

Liu J., V. Angelopoulos, A. Runov and X.-Z. Zhou (2014), Magnetic flux transport by dipolarizing flux bundles, J. Geophys. Res., 119, 909-926, doi:10.1002/2013JA019395.

This article is protected by copyright. All rights reserved. 
T. Y. Lui, C.-L. Chang, A. Mankofsky, H.-K. Wong, D. Winske (1991), A crossfield current instability for substorm expansions, J. Geophys. Res., 96, A7, 11,389-11,401

Lui A.T.Y., Current disruption in the Earth's magnetosphere: Observations and models (1996), J. Geophys. Res., 101, 13,067.

Lui A.T.Y. (2004), Potential plasma instabilities for substorm expansion onsets, Space Sci. Rev., 113, 1, 127-206, 10.1023/B:SPAC.0000042942.00362.4e.

A. T. Y. Lui (2011), Reduction of the cross-tail current during near-Earth dipolarization with multisatellite observations, J. Geophys. Res., 116, A12239, doi:10.1029/2011JA017107.

A.T.Y. Lui (2016), Cross-field current instability for auroral bead formation in breakup arcs, Geophys. Res. Lett., 43, 6087-6095, doi:10.1002/2016GL069892.

Nakamura et al. (2009), Evolution of dipolarization in the near-Earth current sheet induced by Earthward rapid flux transport, Ann. Geophys., 27, 1743.

Nakamura et al. (2013), Flow bouncing and electron injection observed by Cluster, J. Geophys. Res., 118, 5, 2055-2072, doi:10.1002/jgra.50134.

Nakamura, R., Sergeev, V. A., Baumjohann, W., Plaschke, F., Magnes, W., Fischer, D.,

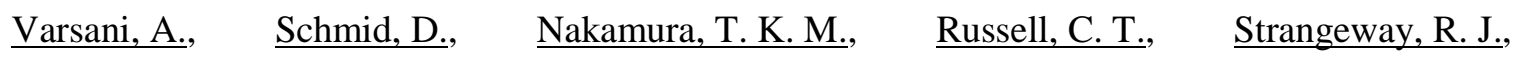
Leinweber, H. K., $\quad$ Le, G., $\quad$ Bromund, K. R., $\quad$ Pollock, C. J., $\quad$ Giles, B. L., $\quad$ Dorelli, J. C., Gershman, D. J., Paterson, W., Avanov, L. A., Fuselier, S. A., Genestreti, K., Burch, J. L., Torbert, R. B., Chutter, M., Argall, M. R., Anderson, B. J., Lindqvist, P.-A., Marklund, G. T., Khotyaintsev, Y. V., $\quad$ Mauk, B. H., $\quad$ Cohen, I. J., $\quad$ Baker, D. N., $\quad$ Jaynes, A. N., Ergun, R. E., 
Singer, H. J., Slavin, J. A., Kepko, E. L., Moore, T. E., Lavraud, B., Coffey, V., Saito, Y. (2016), Transient, small-scale field-aligned currents in the plasma sheet boundary layer during storm time substorms, Geophys. Res. Lett., 43, 10, 4841-4849, doi: 10.1002/2016GL068768.

Ohtani S., M.A. Shay, T. Mukai (2004), Temporal structure of the fast convective flow in the plasma sheet: Comparison between observations and two-fluid simulations, J. Geophys. Res., 109, 3210, doi:10.1029/2003JA010002.

Panov E.V. et al. (2010), Multiple overshoot and rebound of a bursty bulk flow, Geophys. Res. Lett., 37, L08103, doi:10.1029/2009GL041971.

E.V. Panov, R.A. Wolf, M. V. Kubyshkina, R. Nakamura, W. Baumjohann (2015), Anharmonic oscillatory flow braking in the Earth's magnetotail, Geophys. Res. Lett., 42, 3700-3706, doi:10.1002/2015GL064057.

Réme, H., C. Aoustin, J. M. Bosqued, I. Dandouras et al. (2001), First multispacecraft ion measurements in and near the Earth's magnetosphere with identical Cluster ion spectrometry (CIS) experiment, Ann. Geophys., 19, 1303.

Roux A., et al. (1991), Plasma sheet instability related to the westward traveling surge, J. Geophys. Res., 96, 17,697.

Runov A., V. Angelopoulos, M.I. Sitnov, V.A. Sergeev, J. Bonnell, J.P. McFadden, D. Larson, K. Glassmeier, U. Auster (2009), THEMIS observations of an earthward-propagating dipolarization front, Geophys. Res. Lett., 36, L14106, doi:10.1029/2009GL038980.

This article is protected by copyright. All rights reserved. 
Runov A., V. Angelopoulos, X.-Z. Zhou, X.-J. Zhang, S. Li, F. Plaschke, J. Bonnell (2011), A THEMIS multicase study of dipolarization fronts in the magnetotail plasma sheet, J. Geophys. Res., 116, A052216,doi:10.1029/2010JA016316.

Sergeev V., Angelopoulos V., Apatenkov S., Bonnell J., Ergun R., Nakamura R., McFadden J., Larson D., Runov A. (2009), Kinetic structure of the sharp injection/dipolarization front in the flow-braking region, Geophys. Res., Lett., 36, L21105, doi:10.1029/2009GL040658.

V. A. Sergeev, V. Angelopoulos, and R. Nakamura (2012), Recent advances in understanding substorm dynamics, Geophys. Res. Lett., 39, L05101, doi:10.1029/2012GL050859.

Shiokawa, K., W. Baumjohann, and G. Haerendel (1997), Braking of high-speed flows in the near-Earth tail, Geophys. Res. Lett., 24(10), 1179-1182.

Sönnerup, B.U.Ö., M. Scheible. in: Analysis Methods for Multi-Spacecraft Data, ed. G. Pashmann \& P.W. Daly. 1998. ISSI Scientific Report SR-001. Bern, Chap. 8. P. 185.

Zhou M., M. Ashour-Abdalla, X. Deng, D. Schriver, M. El-Alaoui, Y. Pang (2009), THEMIS observation of multiple dipolarization fronts and associated wave characteristics in the near-Earth magnetotail, Geophys. Res. Lett., 36, L20107,doi:10.1029/2009GL040663.

This article is protected by copyright. All rights reserved. 


\section{Figure captions}

Figure 1. An overview of dipolarization observed on 20 July 2013. From top to bottom are shown: $A L$ index; the $|B| ; B_{Z}$ and $B_{X}$ from four Cluster (a-d); three components of proton bulk velocity from $\mathrm{C} 4(\mathbf{e})$; the dawn-dusk component of electric field, $E_{Y}$, from four Cluster (f); electron density, $N_{\mathrm{e}}$, from C3 (g); the $\Delta B_{X} / \Delta Y_{\mathrm{C} 3-\mathrm{C} 4}(\mathbf{h})$ and the $\Delta B_{X} / \Delta Y_{\mathrm{C} 1-}$ $\mathrm{C}_{4}$ (i). The grey profiles display the $\Delta B_{X} / \Delta Y$ including the background level, while the black profiles show only the $\Delta B_{X} / \Delta Y$ values which exceed the background level.

Figure 2. In the left: a zoom of the pink shaded interval shown in Figure 1. From top to bottom are shown: the $B_{Z}$ field (a) and electron density, $N_{e}$, from $\mathrm{C} 3$ (b); the $\Delta B_{X} / \Delta Y_{\mathrm{C} 3-\mathrm{C} 4}$ (c); three components of proton bulk velocity (d) and the dawn-dusk electric field, $E_{Y}$, from C4 (e). Three intervals (I-III) of strong $\Delta B_{X} / \Delta Y_{\mathrm{C} 3-\mathrm{C} 4}$ variations are shaded in gray. In the right part of the Figure the spatial profiles of the electric current density $J_{Z}{ }^{*}$ estimated for intervals I - III are shown.

Figure 3. (a-d): The results of epoch superposition analysis applied to 14 dipolarization events. The absolute values of electric current density $\left|J_{M}{ }^{*}\right|$ are scaled according to the right vertical axis of Figure 3b,c. (e): A histogram of the occurrence frequency of strong $\left|\Delta B_{L} / \Delta N_{\mathrm{C} 3-\mathrm{C} 4}\right|$ gradients with a given duration. (d): A histogram of the probability distribution of $\left|\Delta B_{L} / \Delta N_{\mathrm{C} 3-\mathrm{C} 4}\right|$ and the corresponding $\left|J_{M}{ }^{*}\right|$. Values of $\left|\Delta B_{L} / \Delta N_{\mathrm{C} 3-\mathrm{C} 4}\right|$ are 
binned according to the bottom horizontal axis, and the $\left|J_{M}{ }^{*}\right|$ are given in the upper horizontal axis. 

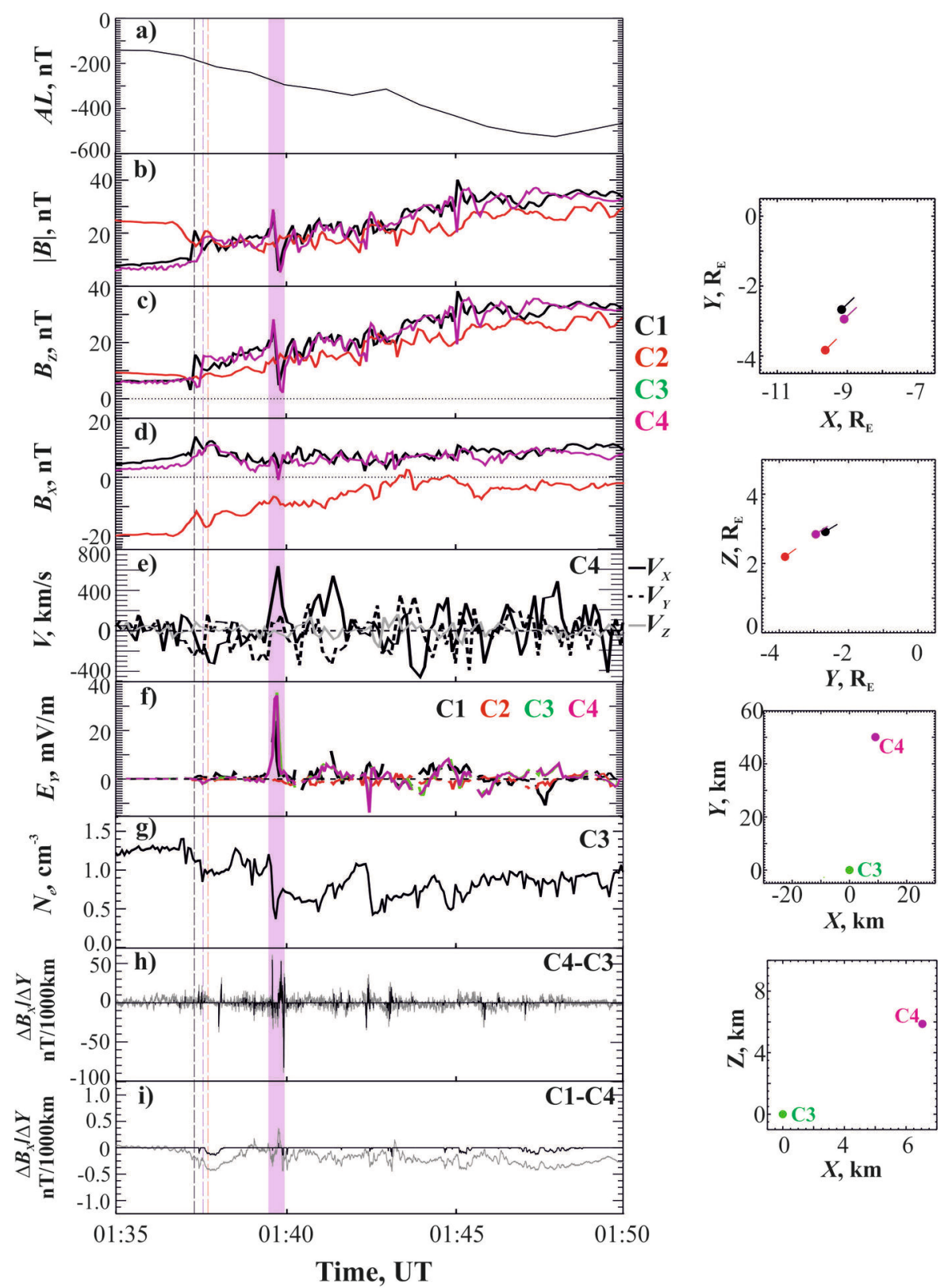

Figure 1.

2017GL076303-f01-z-jpg 

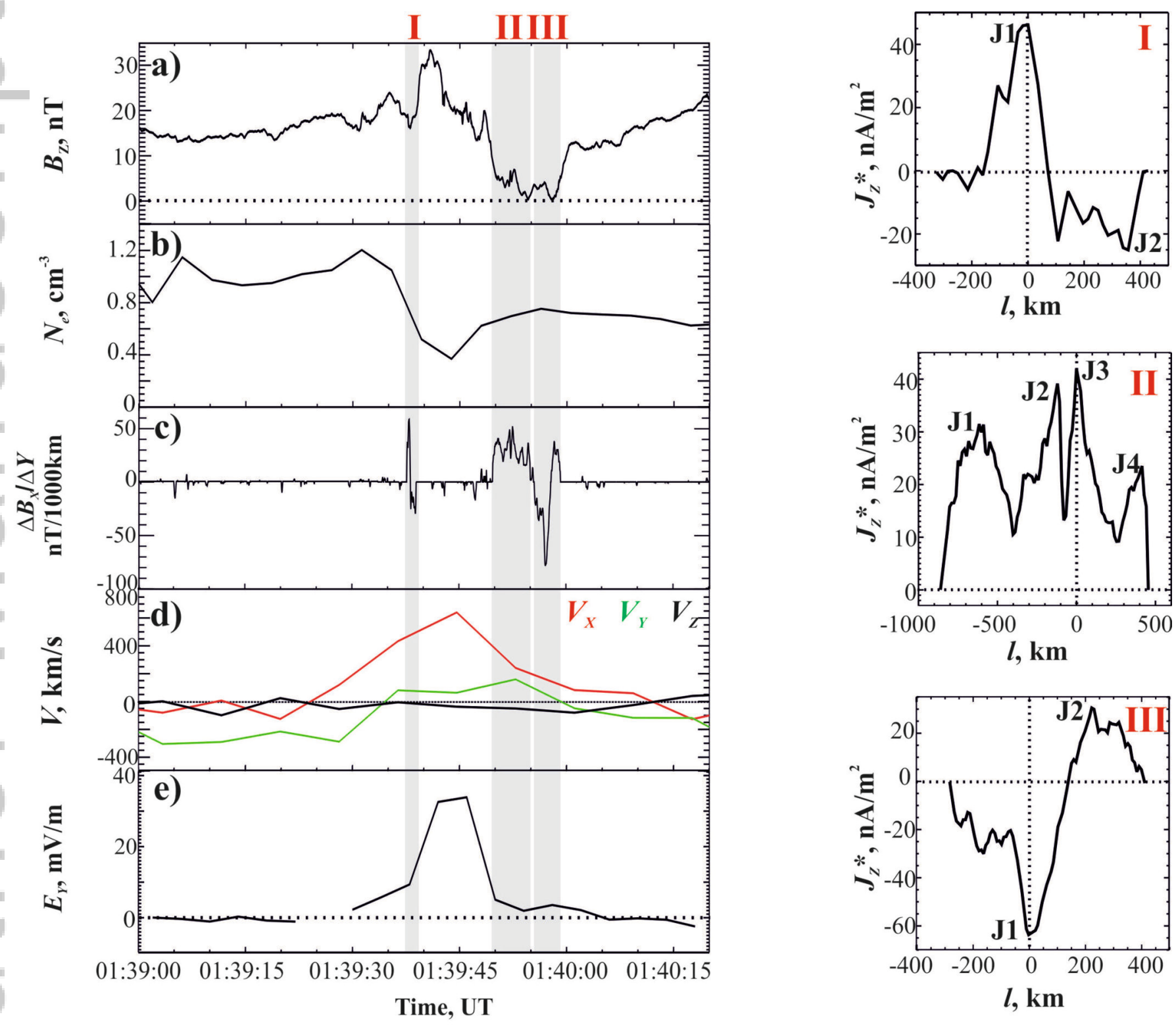

Figure 2.

2017GL076303-f02-z-.jpg 

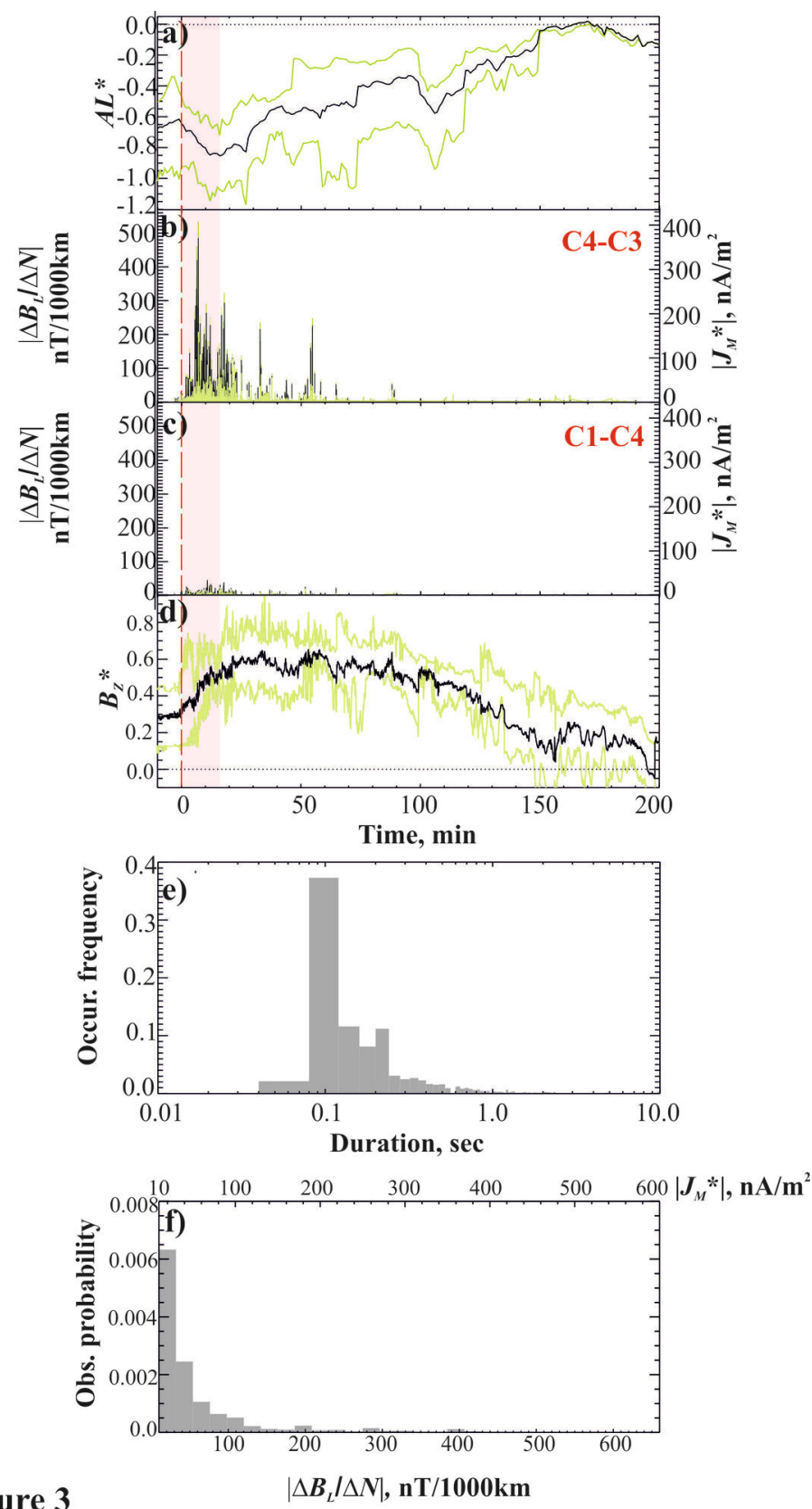

Figure 3

$$
\text { 2017GL076303-f03-z-.jpg }
$$

